\title{
Numerical Simulation of Gas-Liquid Flow in a Bubble Column by Intermittent Aeration in Newtonian Liquid/Non-Newtonian Liquid
}

\author{
Zheng Xipeng, Wang Le, Jia Xiaoxuan, Xiang Wenchuan, and Yang Shunsheng $\mathbb{1}$ \\ Southwest Jiaotong University, School of Civil Engineering, Cheng du, China \\ Correspondence should be addressed to Yang Shunsheng; seanse@126.com
}

Received 25 June 2018; Accepted 13 September 2018; Published 6 November 2018

Academic Editor: Doraiswami Ramkrishna

Copyright (c) 2018 Zheng Xipeng et al. This is an open access article distributed under the Creative Commons Attribution License, which permits unrestricted use, distribution, and reproduction in any medium, provided the original work is properly cited.

\begin{abstract}
The dynamic behaviors of gas-liquid two-phase flow were simulated in a lab-scale intermittent bubble column by Euler-Euler twofluid model coupled with the PBM (population balance model) using two different liquid phases, i.e., Newtonian fluid (water)/non-Newtonian fluid (activated sludge). When non-Newtonian fluid was used during intermittent aeration, some interesting results were obtained. Two symmetric vortexes existed in the time-averaged flow field; the vertical time-averaged velocity of the liquid phase decreased with increasing anaerobic time; the average gas holdup distribution was like a trapezoid with long upper side and short lower side and affected by the dynamic viscosity of the liquid phase. Compared with non-Newtonian fluid, the use of Newtonian fluid as the liquid phase led to a more complicated time-averaged flow field structure and vertical timeaveraged velocity distribution, higher average gas holdup, and the asymmetric column-shaped gas holdup distribution with increasing anaerobic time. For different liquid phases, the instantaneous flow field, instantaneous vertical velocity, and instantaneous gas holdup distribution all periodically changed with anaerobic time; however, different from Newtonian liquid phase, non-Newtonian liquid phase had no periodic oscillating instantaneous horizontal velocity.
\end{abstract}

\section{Introduction}

As an aeration reactor, the bubble column is widely used for study on wastewater treatment because of its low price, easy transport, and high mass transfer characteristics [1-4]. The bubble column is mainly researched by experiment and numerical simulation $[5,6]$. Numerical simulation is extensively used for study on bubble column due to its good economics and ability to obtain the information in the entire bubble column, such as velocity distribution, gas phase distribution, and turbulence energy. Ali et al. [7, 8] used experiments and numerical simulation method for numerical simulation of a rectangular bubble column. For numerical simulation, they considered the effects of different drag models, simulation methods, and model dimensions on the simulation results. The results showed that different drag models did not significantly influence the predicted velocity, and Euler-Euler approach had better simulation results than
Euler-Lagrangian approach. Wang et al. [9] used CFD-PBM for simulation of the bubble column, and their results showed that this model was effective for prediction of bubble size distribution, interfacial area, gas-liquid mass transfer rate, etc., in the bubble column. Gupta and Roy [10] used the PBM for numerical simulation of a rectangular bubble column by considering bubble breakage and coalescence, analysis of different breakage and coalescence equations, and the effects of lift force and virtual mass force on the flow field and gas content in the bubble column.

Pure water in Newtonian fluids is always used as the liquid phase in the above simulations in bubble columns, but few researchers are studying the activated sludge (nonNewtonian fluid). It should be noted that the liquid phase is activated sludge when the bubble column is used as a sewage treatment facility. Activated sludge is not transparent, so it is very difficult to efficiently test the flow field in the bubble column by particle image velocimetry (PIV) and 
laser Doppler velocimetry (LDV) and to obtain the flow regime and flow characteristics in the bubble column. Fan et al. [11] used polystyrene spheres instead of activated sludge to experimentally study the lab-scale oxidation ditch by PDA (particle dynamic analyser) but did not take the activated sludge as non-Newtonian fluid into account $[12,13]$. For the rheological behavior of a liquid, transparent Newtonian fluids, such as aqueous solution of sodium carboxymethyl cellulose (CMC) or Xanthan solution, always were experimentally used as the liquid phase [14-16]. Passos et al. [17] additionally used the nonionic surface active agent Triton X-100 to modify the surface tension of the nonNewtonian solutions and found that the diameter of the bubbles decreased. Dapelo et al. [18] used CMC as a liquid for numerical simulation of the anaerobic digestion unit based on the Euler-Lagrangian approach and found that the relative simplicity of the viscosity model did not affect the results of the simulations. Bandyopadhyay and Das [19] used the non-Newtonian pseudoplastic power law model to simulate the flow through elbows under non-Newtonian liquid and obtained an ideal result. $\mathrm{Wu}$ [20] performed the gas-liquid numerical simulation of an anaerobic digester and considered the effect of non-Newtonian liquid. His results showed that the low Reynolds number $k-\varepsilon$ model was better than other turbulence models, and the gas-liquid mixing efficiency depended on the mixing mechanism and pumping cycle, but he used the drag force model for Newtonian fluid. There was a difference in drag force equation between Newtonian and non-Newtonian fluids [21], so the simulation results would be evaluated.

However, most liquid phases for numerical simulation of gas-liquid two-phase flow in a bubble column are Newtonian fluids, and there is a rare literature on dynamic behaviors of gas-liquid two-phase flow of Newtonian/nonNewtonian liquid phase in an intermittent aerating bubble column. Thus, Euler-Euler two-fluid model coupled with PBM (EEPBM) was used in this paper for numerical simulation of gas-liquid two-phase flow in a lab-scale bubble column and analysis of dynamic behaviors of the fluids in a bubble column, such as gas holdup distribution, liquidphase flow field, and liquid phase velocity field, based on the comparison with the experiment [22] and verification of the mathematical model. The drag force equation and intermittent aeration control of the non-Newtonian fluids were simulated by UDF (user-defined program). The research results provide references and guides for optimized design of the intermittent aerating bubble column for wastewater treatment.

\section{Mathematical Model}

An Euler-Euler two-fluid model (EE) was used to simulate gas-liquid two-phase flow, and PBM was considered to simulate the bubble coalescence and breakage. During the simulations, the gas-liquid interphase heat transfer was ignored, and the two phases were considered for the incompressible fluids. We adopted the assumptions that the mixture of the activated sludge and water was a single-phase liquid [23].
2.1. Euler-Euler Two-Fluid Model. Mass conservation equation:

$$
\frac{\partial}{\partial t}\left(\alpha_{q} \rho_{q}\right)+\nabla \cdot\left(\alpha_{q} \rho_{q} u_{q}\right)=0 .
$$

Momentum conservation equation:

$\frac{\partial}{\partial t}\left(\alpha_{q} \rho_{q} u_{q}\right)+\nabla \cdot\left(\alpha_{q} \rho_{q} u_{q} u_{q}\right)=-\alpha_{q} \nabla p+\nabla \cdot \tau_{q}+\alpha_{q} \rho_{q} g+F$,

where $\alpha$ is the volume fraction; $\rho$ is the density, $\mathrm{kg} / \mathrm{m}^{3} ; \tau$ is the shear stress, $\mathrm{Pa}$, determined from Equation (8); $p$ is the pressure, $\mathrm{Pa} ; g$ is the acceleration of gravity, $9.8 \mathrm{~m} / \mathrm{s}^{2} ; q$ is the phase division, with $g$ being the gas phase and $l$ being the liquid phase; $F$ is the two-phase interphase force, the drag and lift forces were considered in this paper [10]. The twophase interphase force is calculated as follows:

$$
F=F_{\mathrm{D}}+F_{1} \text {. }
$$

The drag force $F_{\mathrm{D}}$ and the lift force $F_{1}$ were taken into account in this work. The lift force is calculated as follows:

$$
F_{l}=C_{l} \alpha_{g} \rho_{l}\left(u_{g}-u_{l}\right) \times \nabla u_{g},
$$

where $C_{l}$ is the lift coefficient, 0.5 [24].

Drag force can be determined as follows:

$$
F_{D}=\frac{3}{4} \alpha_{g} \alpha_{l} \rho_{l} \frac{C_{D}}{d}\left|u_{g}-u_{l}\right|\left(u_{g}-u_{l}\right)
$$

where $d$ is the bubble diameter. The drag force coefficient model $C_{D}$ can be expressed as follows considering the effects of the rheological properties on non-Newtonian fluids [25]:

$$
C_{D}= \begin{cases}\frac{16}{\mathrm{Re}_{t}}\left(1+0.173 \mathrm{Re}_{t}^{0.657}\right)+\frac{0.413}{1+16300 \mathrm{Re}_{t}^{-1.09}}, & \mathrm{Re}_{t}<135, \\ 0.95, & \mathrm{Re}_{t}<135 .\end{cases}
$$

Since Newtonian and non-Newtonian fluids exhibit different flow behaviors, the definition of Reynolds number for Newtonian fluids is invalid for non-Newtonian fluids [26]. Thus, the Reynolds number of spherical bubbles of non-Newtonian fluids $\mathrm{Re}_{t}$ is calculated as follows [27]:

$$
\operatorname{Re}_{t}=\frac{\rho_{l} d^{n} u_{t}^{2-n}}{K}
$$

where $K$ is the viscosity coefficient, $\mathrm{kg} /(\mathrm{m} \cdot \mathrm{sn}), 0.0741$ and $n$ is the rheological index, 0.49 . The formula for calculating the drag force between gas and liquid in Newton's liquid phase is shown in reference [28].

Turbulent effects were modeled by the RNG $k-\varepsilon$ model. This turbulence model was usually applied to predict the liquid flow pattern and gas holdup at low superficial gas velocity due to its simplest algorithm and lower computational cost $[5,29]$.

2.2. Population Balance Model. In order to study the bubble breakup and coalescence phenomena in the bubble column, 
the population balance model can be applied to calculate the bubble size distribution. According to the researchers' studies $[30,31]$, the population balance equation can be expressed as follows:

$$
\begin{aligned}
\frac{\partial N(v, t)}{\partial t}+\nabla \cdot\left(u_{i} N(v, t)\right) & =S_{i}, \\
S_{i} & =\left\{B_{i}^{+}-B_{i}^{-}+C_{i}^{+}-C_{i}^{-}\right\},
\end{aligned}
$$

where $N(v, t)$ is the bubble size distribution function; $B^{+}, B^{-}$, $C^{+}$, and $C^{-}$are the birth due to coalescence, death due to aggregation, birth due to breakage, and death due to breakage, respectively; and $u_{i}$ is the average velocity of the $i$ th bubble group, $\mathrm{m} / \mathrm{s}$. The discrete method was used to solve [32] Equation (8). The simulations of the breakup phenomena were performed using the Luo and Svendsen [31] model. The model proposed by Luo [33] I was used for the modeling of the coalescence processes. The coalescence and breakage model described in this paper has been used to study the numerical simulation of a bubble column $[5,10,22]$. The number of cases, $i=1$ to 10 , represents the 10 groups of bubbles [22].

\section{Modeling and Calculation}

3.1. Physical Model and Grid. Ali et al. [7] found that 2-D and 3-D models for bubble columns had the same predicted velocity, and 2-D model could reflect the gas-liquid twophase flow behaviors in the bubble column. Thus, a 2-D model was created, as shown in Figure 1(a), with length = $0.45 \mathrm{~m}$ and width $=0.2 \mathrm{~m}$. The gas inlet is modeled as a rectangle area with the length of $0.018 \mathrm{~m}$ at the bottom of the domain in the center, representing the experimental sparger setup. As shown in Table 1 and Figure 1(c), we modeled fourteen different cases according to the distance from the gas inlet center to the bottom center of the bubble column as well as the liquid properties. In the simulation, the air density was set to $1.29 \mathrm{~kg} / \mathrm{m}^{3}$, water density $1000 \mathrm{~kg} / \mathrm{m}^{3}$, viscosity $0.001787 \mathrm{~Pa} * \mathrm{~s}$, and gas-liquid surface tension $0.072 \mathrm{~N} / \mathrm{m}$.

3.2. Calculation Case. Among 14 different intermittent aeration calculation cases as shown in Table 1, Cases 1-7 were for Newtonian liquids (pure water), and Cases 8-14 for non-Newtonian liquids (activated sludge). Cases 1, 2, and 7 had the same flow and ratio of aeration time to anaerobic time, while Cases $3,4,5$, and 6 had different ratios of aeration time to anaerobic time. The aerating gas-phase velocity was $0.0024 \mathrm{~m} / \mathrm{s}$, and the gas velocity was $0 \mathrm{~m} / \mathrm{s}$ in the anaerobic time. To describe the intermittent aeration more accurately, Case 1 was taken as an example. The gas-phase velocity was $0.0024 \mathrm{~m} / \mathrm{s}$ and $0 \mathrm{~m} / \mathrm{s}$ at $40 \mathrm{~s}-43 \mathrm{~s}$ and $43 \mathrm{~s}-46 \mathrm{~s}$, respectively, a cycle was $6 \mathrm{~s}$, and the ratio of aeration time to anaerobic time was $1: 1$. The velocity was changed over time by UDF. It was noted that this paper aimed to describe the dynamic behaviors in a bubble column in the aeration time and anaerobic time. Based on the calculated amount, the set aeration time and anaerobic time were short.

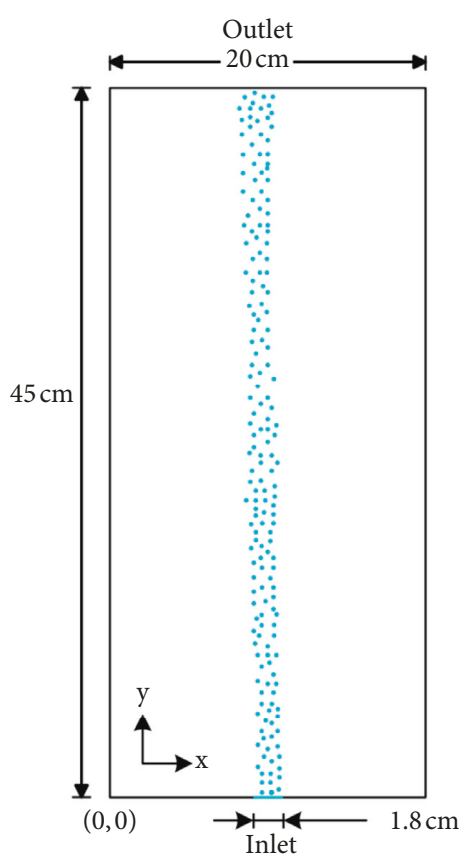

(a)

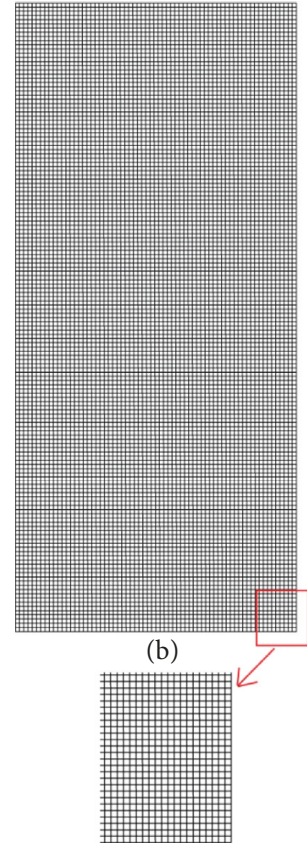

(c)
Figure 1: The physical model configuration and mesh.

3.3. Calculation. The finite volume method was used for the discretization of the equations in Section 1; the volume fractions were differentiated by QUICK pattern; the time item was treated by a first-order scheme; other items were treated via a 2-order upwind discretization scheme; the pressure and velocity were coupled via the Semi-Implicit Method for Pressure-Linked Equation (SIMPLE); the inlet was set to a single-gas phase. The velocity inlet boundary conditions were used, with superficial gas velocity of $0.0024 \mathrm{~m} / \mathrm{s}$; the outlet was set to a gas boundary condition $[34,35]$, while other physical boundaries were set to solidwall boundary conditions. The residual error was set to $10^{-5}$, with a time step length of $0.01 \mathrm{~s}$; the maximum iteration number of 30. 1,000 steps were calculated at steady state first, and then the calculation was performed under continuous gas supply at nonsteady state for $40 \mathrm{~s}$. The data during the $40 \mathrm{~s}$ were used as initial field. The intermittent aeration lasted from $40 \mathrm{~s}$ to $220 \mathrm{~s}$. In this paper, FLUENT 15.0 was used to simulate the example.

\section{Validation}

4.1. Mesh Validation. As for the physical model of Case 1 in Table 1 at four different grid numbers, we simulated the aeration of pure water (Newtonian fluid) and obtained the simulation data about the global gas holdup in the bubble column. In Table 2, the maximum iteration number and time step length at each step were discussed.

As shown in Table 1, the time step length and maximum iteration number both affected the simulation results. The differences in gas holdup between simulation and experiment were proved to be quite similar with the mesh number of 10050 . When the mesh number rose to 22725 , the 
TABle 1: Parameters for different calculation cases.

\begin{tabular}{lcccccc}
\hline Calculation case no. & 1 & 2 & 3 & 4 & 5 & 6 \\
\hline Aeration time (s) & 3 & 6 & 6 & 6 & 6 & 6 \\
Anaerobic time (s) & 3 & 6 & 1.5 & 4 & 9 & 14 \\
Ratio of aeration time to anaerobic time & $1: 1$ & $1: 1$ & $4: 1$ & $3: 2$ & $2: 3$ & $2: 7$ \\
Liquid property & & & Newtonian & $1: 1$ \\
\hline
\end{tabular}

TABLE 2: Global gas holdup with different parameters.

\begin{tabular}{lccc}
\hline Element number & $\begin{array}{c}\text { Time step } \\
\text { size (s) }\end{array}$ & $\begin{array}{c}\text { Maximum } \\
\text { iterations }\end{array}$ & Gas holdup* \\
\hline 5763 & 0.01 & 30 & 0.00670 \\
7353 & 0.01 & 30 & 0.00664 \\
10050 & 0.01 & 30 & 0.00692 \\
22725 & 0.01 & 30 & 0.00664 \\
10050 & 0.0025 & 30 & 0.00692 \\
10050 & 0.005 & 30 & 0.00692 \\
10050 & 0.02 & 30 & 0.00695 \\
10050 & 0.01 & 10 & 0.00693 \\
\hline
\end{tabular}

${ }^{*}$ Gas holdup in the experiments was 0.0069 .

simulation results were not improved compared with the experimental data. Some researchers also found this phenomenon, which might be related to the turbulent spectrum $[35,36]$. However, the above validation was still unable to show the comparison between the gas holdup distribution and the experimental results. Thus, further validation about the gas holdup distribution was conducted in Section 4.2.

4.2. Flow Visualization: Experimental versus Simulated Results. The simulations of gas holdup distribution with the above physical model and mathematical method are shown in Figure 1, where the total mesh number was 10050. By comparing with experimental observations as shown in Figure 2, we found the instantaneous type of simulated gas holdup distribution was S-shaped; the gas holdup near the inlet area was maximum value, while the gas holdup near the outlet area was very low. The simulated gas distribution area from the bottom to the top gradually was enlarged, and all the simulated phenomena on gas holdup distribution were consistent with the experimental observations.

To further validate the correctness of the simulation results, the global gas holdup and plume period time obtained from simulations were compared with the experimental results. Besides, we also used the EE to simulate the liquid-gas flow in the bubble column with uniform bubble size in order to validate the advantages of EEPBM. As shown in Table 3, both the errors of gas holdup determined by EEPBM and by experiments were $0.3 \%$, smaller than that by EE (2.6\%). Moreover, the simulated bubble plume oscillation period showed that the EEPBM was superior to EulerEuler two-fluid model, where the error in bubble plume oscillation period was $1.8 \%$ and $3.2 \%$ compared with experimental data, respectively.

Although EEPBM model had small difference in simulation results from the EE model, the EEPBM model took the actually existing bubble coalescence and breakage in

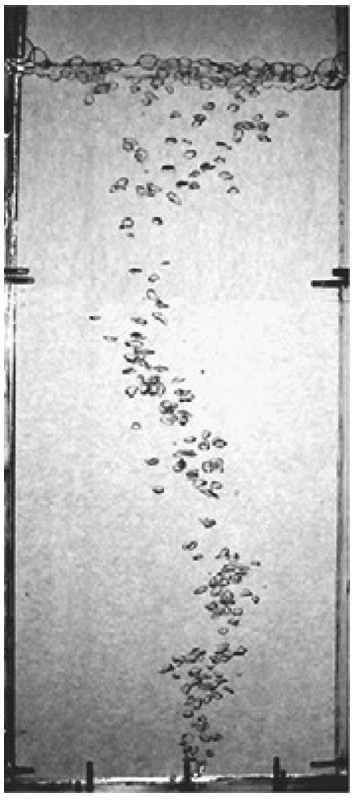

(a)

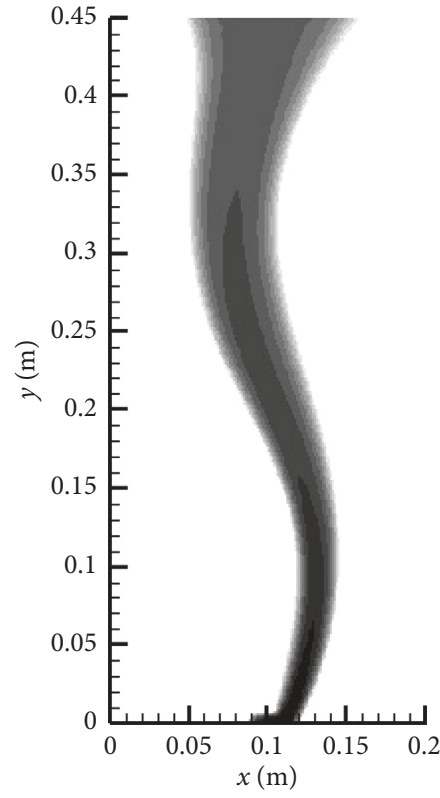

(b)
FIgURE 2: Comparison between experimental and computational results of instantaneous gas holdup.

a bubble column into account. Thus, the EEPBM model was used for calculation in this paper.

\section{Results and Discussion}

5.1. Time-Averaged Liquid-Phase Flow Field and Velocity Field. Figure 3 shows the time-averaged flow field of the liquid phase. A Newtonian fluid was used as the liquid phase in Figures 3(a)-3(g). When the anaerobic time was below $9 \mathrm{~s}$ as shown in Figures 3(a)-3(e), two approximately symmetric main vortexes existed in the bubble column, and their centers were located in the middle lower part of the bubble column. That complied with the time-averaged results from continuous aeration at low velocity given by Díaz et al. [22]. That meant that at a short anaerobic time, the aeration mode did not significantly affect the flow field of Newtonian fluid. When the anaerobic time was above $9 \mathrm{~s}$ as shown in Figures 3(g) and 3(f), the flow field had several asymmetric vortexes in the bubble column and a complicated structure possibly because (1) when the aeration stopped, the liquid phase velocity gradually reduced in the bubble column, (2) the anaerobic time was so short that the liquid phase velocity reduced very little, the flow field could be kept relatively stable, and the vortexes were approximately symmetric in the time-averaged flow field, and (3) on the contrary, when 
TABLE 3: Comparison between experimental and calculated results.

\begin{tabular}{lccccc}
\hline $\begin{array}{l}\text { Measured gas } \\
\text { holdup }\end{array}$ & $\begin{array}{c}\text { Calculated gas } \\
\text { holdup (EEPBM) }\end{array}$ & $\begin{array}{c}\text { Calculated gas } \\
\text { holdup (EE) }\end{array}$ & $\begin{array}{c}\text { Measured plume } \\
\text { oscillation period }\end{array}$ & $\begin{array}{c}\text { Calculated plume } \\
\text { oscillation period (EEPBM) }\end{array}$ & $\begin{array}{c}\text { Calculated plume } \\
\text { oscillation period (EE) }\end{array}$ \\
\hline 0.0069 & 0.00692 & 0.00672 & $11.378(\mathrm{~s})$ & $11.582(\mathrm{~s})$ & $11.738(\mathrm{~s})$ \\
\hline
\end{tabular}

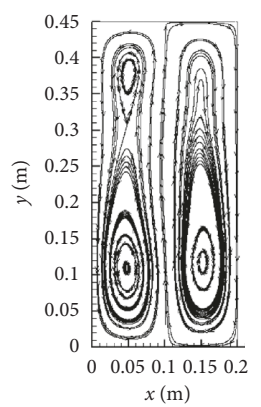

(a)

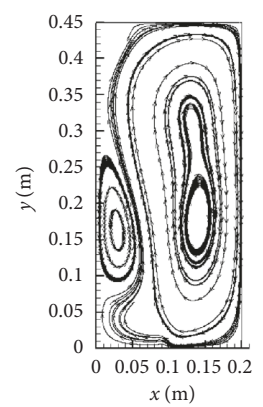

(f)

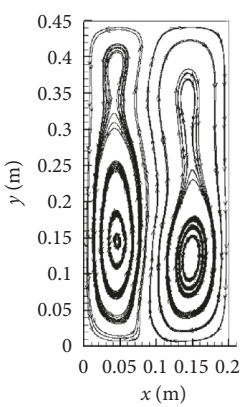

(b)

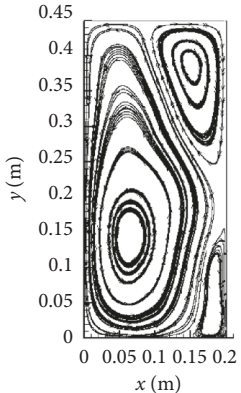

(g)

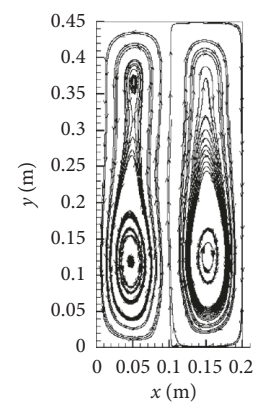

(c)

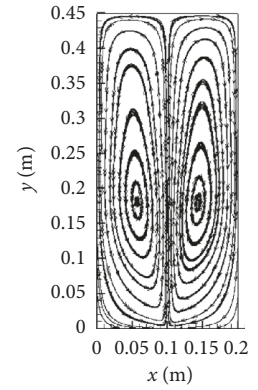

(h)

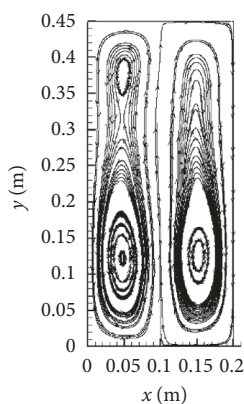

(d)

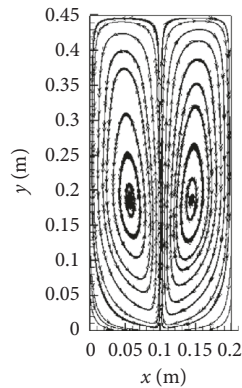

(i)

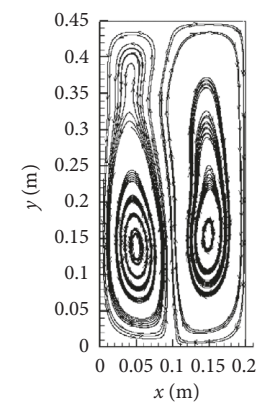

(e)

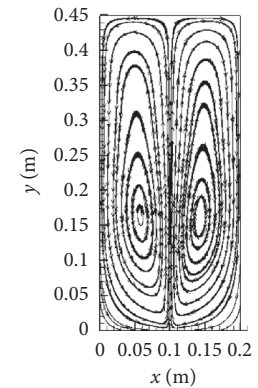

(j)



(k)



(1)

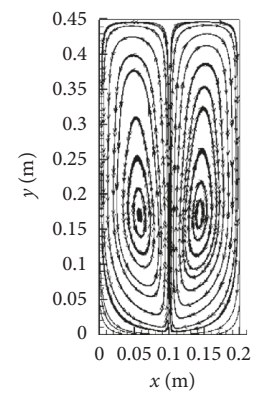

(m)



(n)

Figure 3: Time-averaged flow field of liquid phase. (a) Case 1, (b) Case 2, (c) Case 3, (d) Case 4, (e) Case 5, (f) Case 6, (g) Case 7, (h) Case 8, (i) Case 9, (j) Case 10, (k) Case 11, (l) Case 12, (m) Case 13, and (n) Case 14.

the anaerobic time was long, the velocity reduced very greatly, and the flow field in the bubble column changed complicatedly, resulting in several vortexes, vortexes distribution change, and a complicated structure of the timeaveraged flow field.

Figures $3(\mathrm{~h})-3(\mathrm{n})$ shows the time-averaged flow field of the non-Newtonian fluid as the liquid phase and showed that the flow fields were the same at different ratios of aeration time to anaerobic time and at anaerobic time, i.e., symmetric at $x=0.1 \mathrm{~m}$. The ratio of aeration time to anaerobic time and anaerobic time did not significantly affect the flow field of non-Newtonian fluid as the liquid phase in the bubble column possibly because after affected by rheological behavior of non-Newtonian fluid, the gas went up from the low dynamic viscosity zone of the liquid phase to form an upward path of gas from the bubble column bottom to top, while the liquid phase was driven by the gas phase, resulting in a symmetric swirl in the flow field. The dynamic viscosity of the liquid phase would be further described in Section 5.4.

Figures 4(a) $-4(\mathrm{~d})$ shows the vertical time-averaged velocity-of the liquid phase at the height $y=0.225 \mathrm{~m}$, Figures 4(a) and 4(b) shows the time-averaged velocity distribution of Newtonian fluid, and Figures 4(c) and 4(d) shows the time-averaged velocity distribution of nonNewtonian fluid. It could be found from Figure 4 that the liquid phase was driven by the gas phase to flow up 


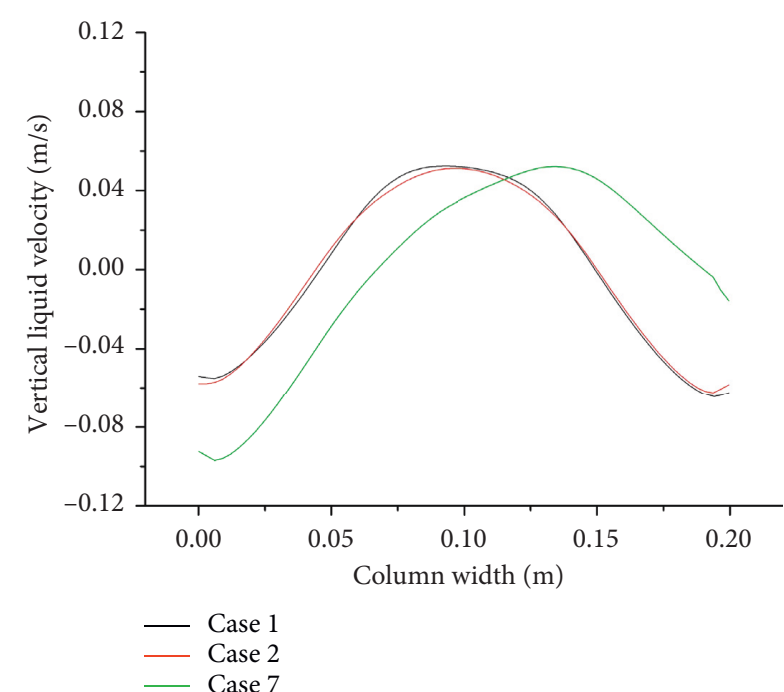

(a)

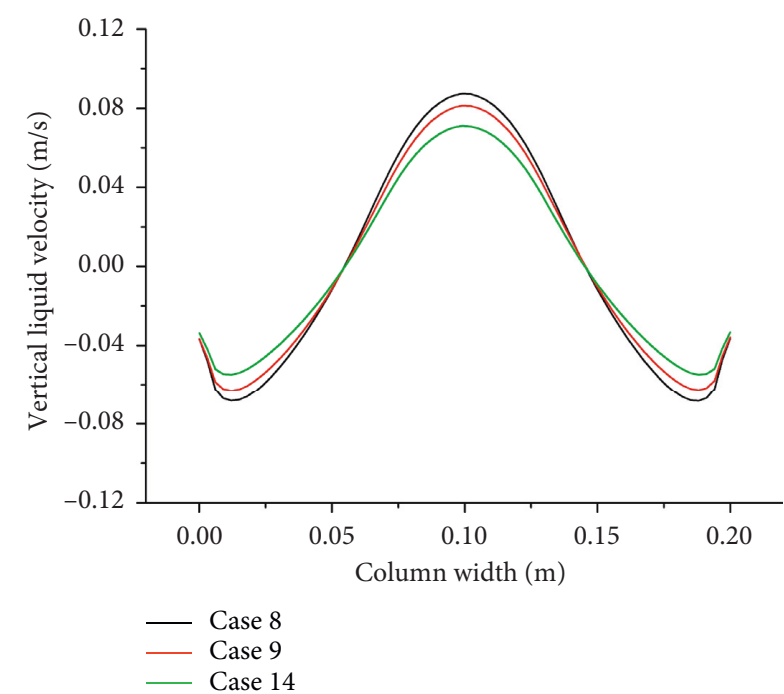

(c)

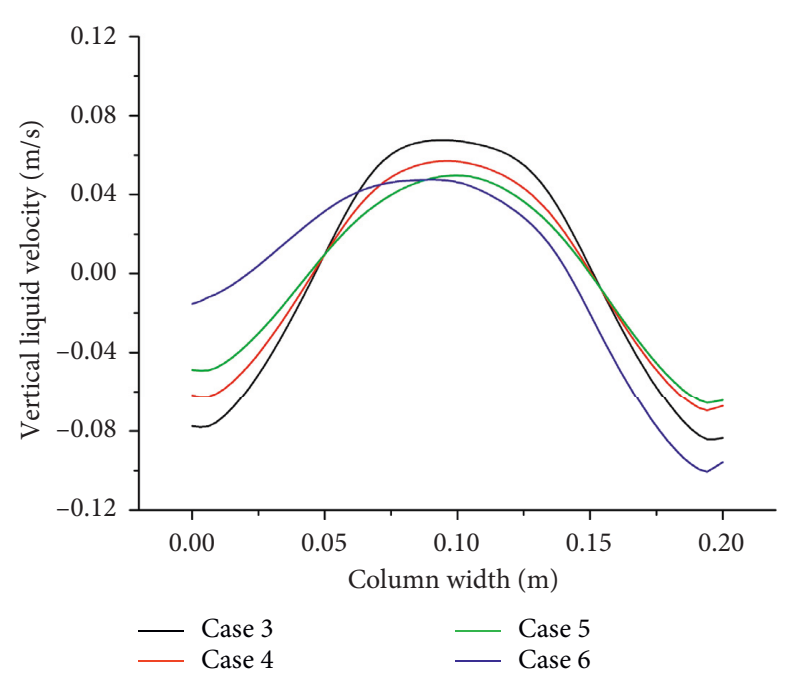

(b)

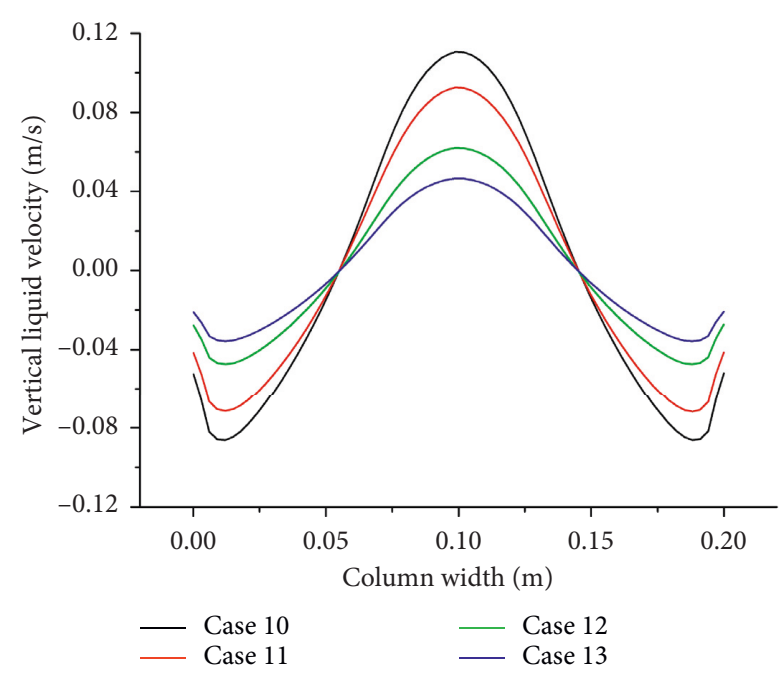

(d)

Figure 4: Vertical time-averaged velocity distribution of liquid phase.

through the column top to the column walls at two sides and finally went back to the column bottom, and the vertical timeaveraged velocity of the liquid phase was big in the middle and small at two sides whether the liquid phase was Newtonian or non-Newtonian fluid. Figure 4(a) shows that at the same ratio of aeration time to anaerobic time, Cases 1,2, and 7 had the same vertical velocity peak value of the liquid phase; however, when the anaerobic time increased, the time-averaged velocity of the liquid phase was much bigger at the left wall than the right wall, and the center where the peak occurred significantly moved to the right because of the impact of flow field structure change. Figure 4(b) shows that when anaerobic time was short at different ratios of aeration time to anaerobic time, the vertical time-averaged velocity of the liquid phase had the approximately symmetric distribution, as shown in Cases 3-5; when the anaerobic time increased, the velocity was much bigger at the right wall than the left wall, as shown in Case 6; with decreasing ratio of aeration time to anaerobic time, the velocity peak value gradually reduced because the amount of aeration reduced.

Figure 4(d) shows that like Newtonian fluid, the velocity peak value of non-Newtonian fluid gradually decreased with decreasing ratio of aeration time to anaerobic time. In Figures 4(c) and 4(d), when the anaerobic time was long, the vertical time-averaged velocity of the liquid phase was still symmetric as shown in Cases 13 and 14. In addition, it was found during comparison of Figures 4(a) and 4(c) and comparison of Figures 4(b) and 4(d) that the liquid phase had much higher vertical time-averaged velocity peak value than Newtonian fluid under the same aeration conditions maybe because the gas phase had smaller distribution area than the Newtonian liquid phase (Section 5.2) at the height $y=0.225 \mathrm{~m}$. This meant that when the gas phase flowed up at a small zone, the time-averaged velocity increased, resulting in a higher time-averaged vertical velocity after the liquid phase was driven. It was found during comparison of 
velocity peak values at the same ratio of aeration time to anaerobic time in Cases 8, 9, and 14 in Figure 4(c) that the shorter the anaerobic time was, the higher the velocity peak value was maybe because when the aeration stopped for a short time, the vertical velocity of the liquid phase decreased very little, and a high vertical velocity could be reached during reaeration. Different from Newtonian fluid, the time-averaged velocity of non-Newtonian fluid had the $\mathrm{V}$-shaped distribution near the walls at two sides of the bubble column maybe because of high dynamic viscosity near the walls (Section 5.4).

5.2. Time-Averaged Gas Holdup Distribution. Figure 5 shows the time-averaged gas holdup distribution. For Newtonian fluid, the gas holdup had the approximately columnar distribution when the anaerobic time was short as shown in Figures 5(a)-5(e). When the anaerobic time was long, the irregular distribution of gas holdup had occurred as shown in Figures $5(\mathrm{f})$ and $5(\mathrm{~g})$. In addition, Figures 5(c)-5(f) showed that with decreasing ratio of aeration time to anaerobic time, both the gas holdup distribution area and the high gas-phase volume fraction area gradually decreased, indicating that the ratio of aeration time to anaerobic time directly affected the gas holdup distribution area. Figures 5(a), 5(b), and 5(g) showed the gas holdup distribution at the same ratio of aeration time to anaerobic time. With increasing anaerobic time, the gas holdup distribution gradually became asymmetric.

Figures 5(h)-5(n) shows non-Newtonian fluid gas volume fraction distribution and showed that the gas phase had the symmetric distribution, i.e., a trapezium with long upper side and short lower side. Figures 5(h), 5(i), and 5(n) shows the same gas holdup distribution at the same ratio of aeration time to anaerobic time. Figures 5(i) and 5(n) had the bigger gas volume fraction distribution area than Figure $5(\mathrm{~h})$, which did not seem to match the gas content because Figures 5(i) and 5(n) had smaller gas volume fraction distribution area than Figure 5(h). Figures $5(\mathrm{j})-5(\mathrm{~m})$ shows the gas-phase distribution at different ratios of aeration time to anaerobic time. With decreasing ratio of aeration time to anaerobic time, the gas-phase distribution area gradually decreased, and the high gas-phase volume fraction area decreased, too. In addition, for non-Newtonian fluid as the liquid phase, the gas-phase volume fraction area had V-shaped distribution in the middle of and in the upper center of the bubble column, while the gas-phase volume fraction at the midzone was low maybe because of high dynamic viscosity of the liquid phase in the middle of the bubble column (Section 5.4).

In summary, the gas holdup distribution was affected by the property of the liquid phase in the bubble column in the same aeration mode. For Newtonian fluid, the gas-phase distribution was approximately columnar. Different from Newtonian fluid, non-Newtonian fluid had the trapezoidal distribution, and the low gas-phase distribution area was very big at the bubble column top.

5.3. Global Gas Holdup and Bubble Diameter. To further analyze the effect of the aeration time and anaerobic time on the gas holdup, the time-averaged global gas holdup in the bubble column was determined. For Newtonian fluid, the gas holdup in a bubble column gradually decreased to a plateau with increasing anaerobic time at the same ratio of aeration time to anaerobic time, as shown in Cases 1, 2, and 7 in Figure 6. The average gas holdup was 0.00344 at the maximum anaerobic time of $10 \mathrm{~s}$ maybe because at the ratio of aeration time to anaerobic time, the shorter anaerobic time, the stronger turbulence energy, the higher gas-liquid mixing intensity, the higher gas holdup. With decreasing ratio of aeration time to anaerobic time, the average gas holdup gradually decreased mainly because the quantity of aeration was reduced. Non-Newtonian fluid had the same gas holdup change as Newtonian fluid. Furthermore, Newtonian fluid had much higher gas holdup than nonNewtonian fluid under the same conditions. This complied with the research results from Durán et al. [37] because of the higher gas velocity in non-Newtonian fluid than Newtonian fluid.

As a main parameter of the bubble column, the bubble diameter was very important to predict the gas-liquid mass transfer in the bubble column. It could be found from Figure 7 that the volume-average bubble diameter was between 0.0054 and $0.0062 \mathrm{~m}$. It was found during comparison of Cases 3-6 at different anaerobic times that for Newtonian liquid, the volume-average bubble diameter gradually reduced because the quantity of aeration reduced. For nonNewtonian liquid, the bubble diameter had no significant difference, and the aeration mode did not significantly influence the volume-average bubble diameter. Moreover, the property of the liquid phase significantly influenced the volume-average bubble diameter, and non-Newtonian liquid had much smaller bubble diameter than Newtonian liquid under the same aeration condition.

5.4. Instantaneous Dynamic Viscosity of Liquid Phase. The bigger dynamic viscosity the fluid had, the weaker flowability the fluid had. The study on the dynamic viscosity distribution in different aeration modes helped further disclose the reason of velocity and gas holdup distribution. Figure 8 showed the dynamic viscosity distribution of the non-Newtonian liquid phase in the bubble column at the aeration time of $162 \mathrm{~s}$ and gave the exponential distribution of dynamic viscosity from less than 0.05 to more than $1.0 \mathrm{~kg} /(\mathrm{m} \cdot \mathrm{s})$; dynamic viscosity peak values were different in different aeration modes; however, the dynamic viscosity peak values were distributed at the bottom of the bubble column and were insignificant to the overall flow in the bubble column and therefore were not discussed herein. The liquid phases had no significant difference in dynamic viscosity distribution in the bubble column. The high dynamic viscosity zone was at two sides of the bubble column bottom, while the low dynamic viscosity zone had the V-shaped distribution and was located at the middle and bottom; the high dynamic viscosity zone close to the wall in the middle of the bubble column had the column barshaped distribution. This meant that the liquid phase had weak flowability and caused V-shaped vertical velocity distribution of the liquid phase near the wall in Figures 4(c) and 4(d). In 


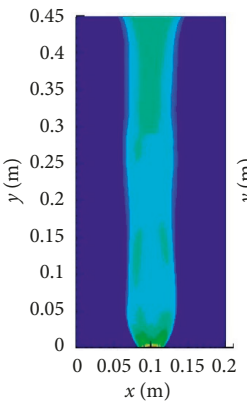

(a)

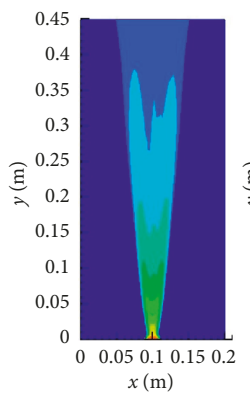

(h)

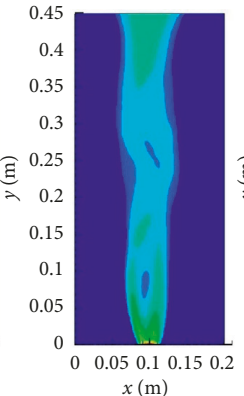

$x(\mathrm{~m})$

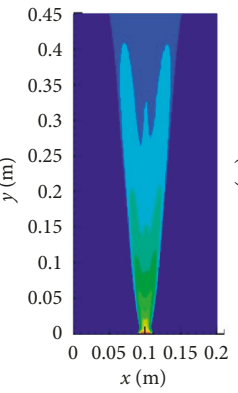

(i)

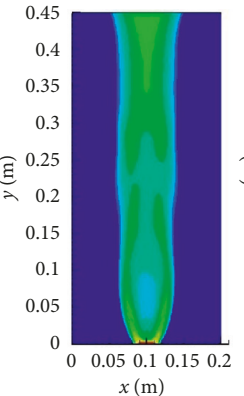

(c)



(j)
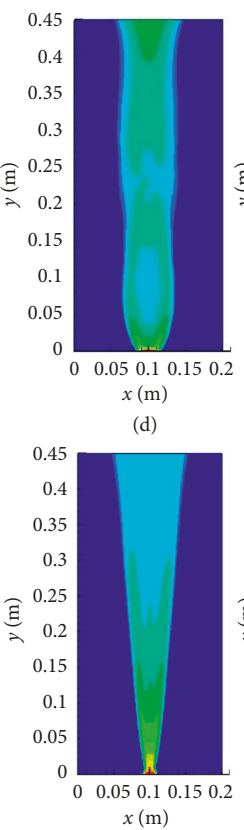

(k)
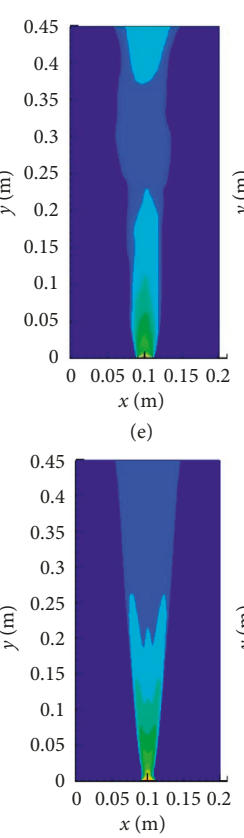

(l)
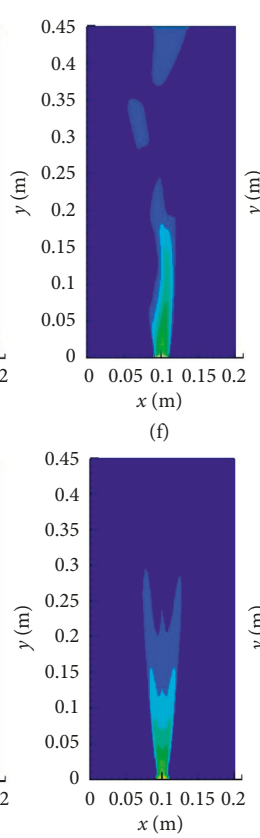

$(\mathrm{m})$
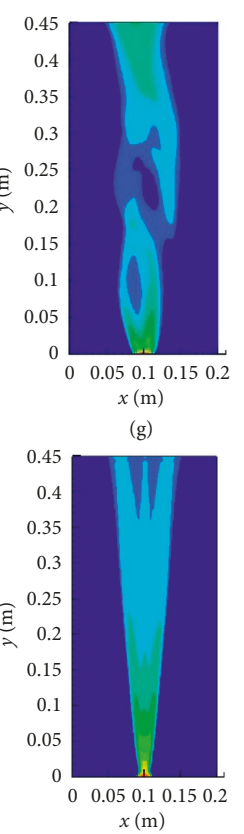

(n)

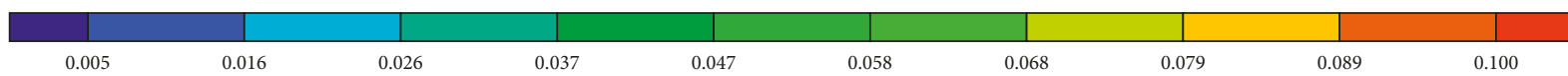

Figure 5: Time-averaged gas holdup distribution. (a) Case 1, (b) Case 2, (c) Case 3, (d) Case 4, (e) Case 5, (f) Case 6, (g) Case 7, (h) Case 8, (i) Case 9, (j) Case 10, (k) Case 11, (l) Case 12, (m) Case 13, and (n) Case 14.

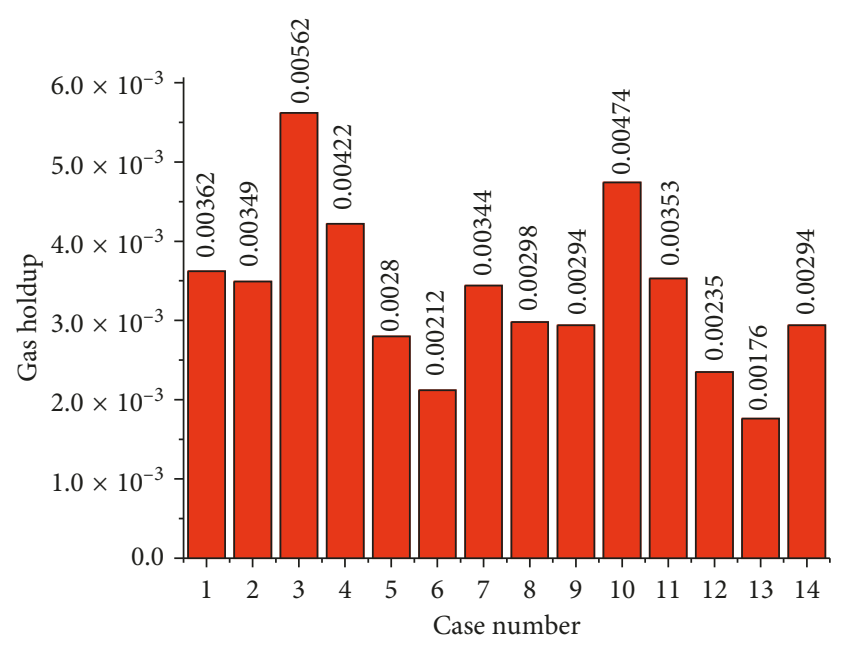

Figure 6: Comparison of gas holdup.

addition, it was found during comparison of Figures $5(\mathrm{~h})-5(\mathrm{n})$ and Figures 8 (a) $-8(\mathrm{~g})$ that low dynamic viscosity zone in the bubble column had the $\mathrm{V}$-shaped distribution; this zone also corresponded to the gas holdup distribution area. This meant that the gas phase always flowed up at the small dynamic viscosity peak value zone.

5.5. Instantaneous Liquid-Phase Velocity and Flow Field. Figure 9 showed the instantaneous liquid-phase flow field in the cycle from 160 to $166 \mathrm{~s}$ in Case 8 . It could be seen from Figure 9 that two main eddies existed in the bubble column and two small symmetric vortexes formed in the middle of



Figure 7: Comparison of volume-average bubble diameter.

the bottom of the bubble column at $160.2 \mathrm{~s}$ because the reinjected gas phase sheared the liquid phase at the bottom. From $161.2 \mathrm{~s}$ to $162.2 \mathrm{~s}$, the gas phase endlessly flowed up to the top of the bubble column and overflowed from the bubble column, so the vortexes at the bottom disappeared and symmetric vortexes formed at both sides of the bubble column. When the gas phase injection stopped, two small eddies formed at both sides of the bubble column bottom at $163.2,164.2$, and $165.2 \mathrm{~s}$ maybe because of the shear action of the liquid phase at both sides of the bubble column, where main vortexes were endlessly compressed due to the big dynamic viscosity of the liquid phase when the liquid phase flowed from the bottom to the middle of the bubble column. 




(a)

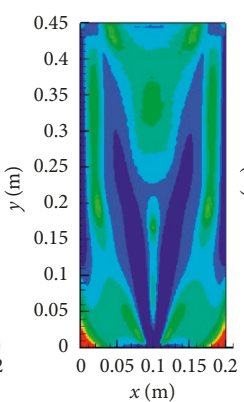

(b)

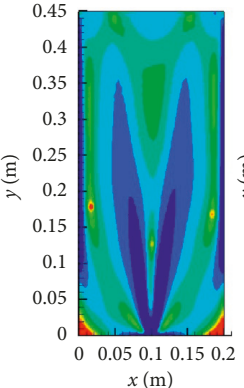

(c)

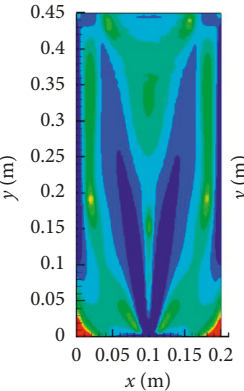

(d)



(e)

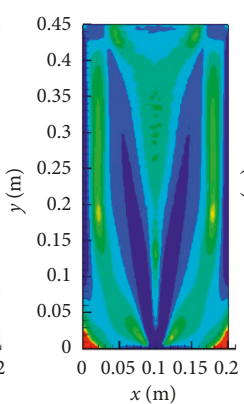

(f)



(g)

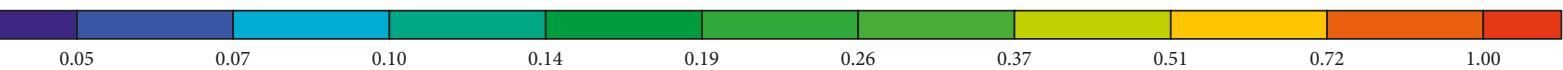

Figure 8: Instantaneous dynamic viscosity distribution of liquid phase. (a) Case 8, (b) Case 9, (c) Case 10, (d) Case 11, (e) Case 12, (f) Case 13 , and (g) Case 14.

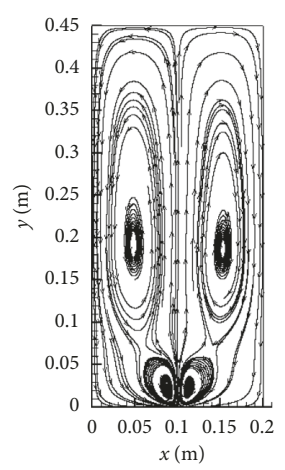

(a)

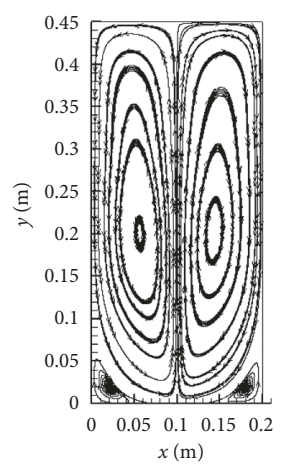

(d)

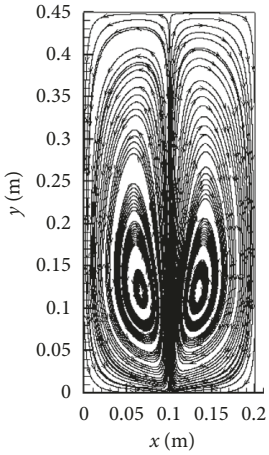

(b)

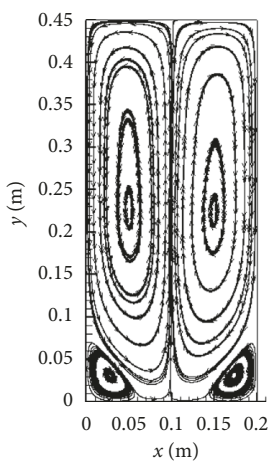

(e)

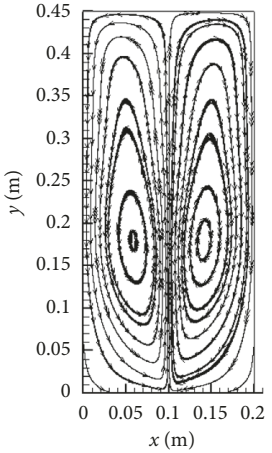

(c)



(f)

FIGURE 9: Instantaneous liquid flow field. (a) $160.2 \mathrm{~s}$, (b) $161.2 \mathrm{~s}$, (c) $162.2 \mathrm{~s}$, (d) $163.2 \mathrm{~s}$, (e) $164.2 \mathrm{~s}$, and (f) $165.2 \mathrm{~s}$.

Figure 10 showed the vertical velocity of the liquid phase in four different calculation cases at the monitoring points in the bubble column, i.e., vertical and horizontal velocity of the liquid phase of Cases 5, 7, 12, and 14 from $100 \mathrm{~s}$ to $220 \mathrm{~s}$. It could be found that for Newtonian fluid, the horizontal velocity in Cases 5 and 7 changed periodically to some different extent, meaning that bubble plume oscillation during continuous aeration also existed during intermittent aeration. For non-Newtonian fluid, the horizontal velocity of the liquid phase in Cases 12 and 14 was constant, meaning that the bubble plume oscillation never happened, which complied with bubble upflow without oscillation in non-Newtonian
(Xanthan solution) observed by Böhm et al. [38]. In addition, the vertical velocity of liquid phases changed periodically in spite of different properties of the liquid phases during intermittent aeration. When aeration began, the vertical velocity of the liquid phases rapidly increased; when the aeration stopped, the axial velocity of the liquid phases rapidly decreased and gradually tended towards $0 \mathrm{~m} / \mathrm{s}$. This cycle had the same time as the intermittent aeration cycle.

5.6. Instantaneous Gas Holdup Distribution. To further study the instantaneous gas holdup distribution for non-Newtonian fluid, Figure 11 shows the gas holdup distribution at the 


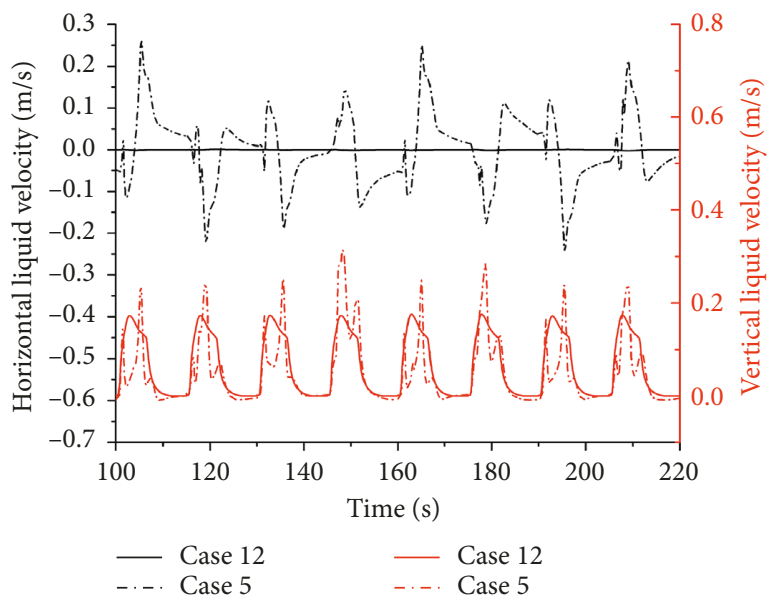

(a)

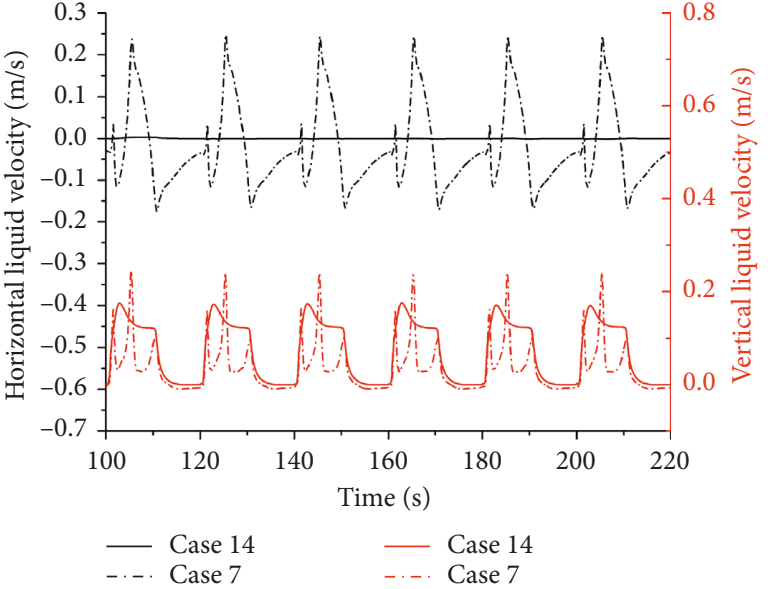

(b)

Figure 10: Instantaneous vertical and horizontal velocity of liquid phase.

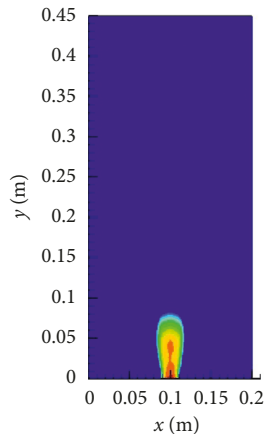

(a)



(b)



(c)



(d)



(e)

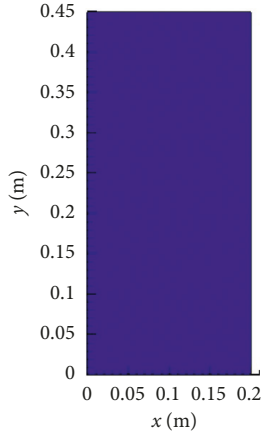

(f)

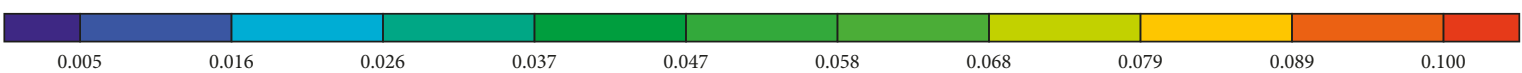

FIGURE 11: Instantaneous gas holdup distribution. (a) $160.2 \mathrm{~s}$, (b) $161.2 \mathrm{~s}$, (c) $162.2 \mathrm{~s}$, (d) $163.2 \mathrm{~s}$, (e) $164.2 \mathrm{~s}$, and (f) $165.2 \mathrm{~s}$.

interval of $1 \mathrm{~s}$ from $160.2 \mathrm{~s}$ to $165.2 \mathrm{~s}$ in the cycle between 160 and $166 \mathrm{~s}$ in Case 8 . Figures 11(a)-11(c) show the gas holdup distribution in the aeration cycle. The gas phase was injected at $160.2 \mathrm{~s}$ and elliptically distributed at the bubble column bottom. From $2 \mathrm{~s}$ to $162.2 \mathrm{~s}$, some of the gas phase overflowed from the top of the bubble column, and the gas-phase distribution was trapezoidal with long upper side and short lower side. The gas phase was stopped at $163 \mathrm{~s}$. No gas holdup distribution at the bottom of the bubble column was observed at $163.2 \mathrm{~s}$, and the same gas holdup distribution in the middle of and in the upper part of the bubble column was observed at $162.2 \mathrm{~s}$. After $1 \mathrm{~s}$, the gas phase completely overflowed from the bubble column. No bubble plume oscillation of the gas phase was observed at any time.

\section{Conclusions}

(1) The intermittent aeration mode of Newtonian fluid affected the time-averaged gas-phase distribution, liquid-phase flow field, and vertical velocity of liquid phase. With increasing anaerobic time, the flow field structure became complicated and the number of vortexes and distribution changed. When the anaerobic time was below $9 \mathrm{~s}$, the vertical velocity distributions of the gas phase and liquid phase were approximately symmetric, and the gas holdup distribution was columnar. The gas holdup slightly decreased with increasing anaerobic time at the same ratio of aeration time to anaerobic time; and the gas holdup decreased with decreasing ratio of aeration time to anaerobic time.

(2) In the intermittent aeration mode, non-Newtonian liquid phase had significant difference in gas holdup distribution, vertical velocity, and flow field from Newtonian liquid phase. The gas-phase distribution was trapezoidal with long upper side and short lower side; liquid phase had much higher vertical velocity peak value than Newtonian fluid in the intermittent aeration mode; the flow field had a simpler structure and did not change with intermittent mode, and 
symmetric vortexes existed at both sides of the bubble column. In the same intermittent aeration mode, non-Newtonian liquid had the same gas holdup change as Newtonian liquid, but nonNewtonian liquid phase had much lower gas holdup than Newtonian liquid phase. For Newtonian liquid phase and non-Newtonian liquid phase, the gas holdup distribution was related to the dynamic viscosity of the liquid phase.

(3) Affected by the intermittent aeration mode, the vertical velocity of Newtonian and non-Newtonian liquid phase changed periodically; however, different from Newtonian liquid phase, non-Newtonian liquid phase had a constant horizontal velocity. For nonNewtonian liquid phase, no bubble plume oscillation appeared in the intermittent aeration mode, and the gas holdup distribution dynamically changed with aeration time. For non-Newtonian liquid phase, the flow field distribution was symmetric in a complete aeration stop cycle; at the beginning of aeration, two small eddies and two main vortexes at the bottom of the bubble column changed to two small eddies and two main vortexes at both sides of the bottom of the bubble column, respectively.

\section{Data Availability}

The data used to support the findings of this study are available from the corresponding author upon request.

\section{Conflicts of Interest}

The authors declare that they have no conflicts of interest.

\section{References}

[1] F. Cerrone, P. Barghini, C. Pesciaroli, and M. Fenice, "Efficient removal of pollutants from olive washing wastewater in bubble-column bioreactor by trametes versicolor," Chemosphere, vol. 84, pp. 254-259, 2011.

[2] J. N. Sahu, S. Agarwal, B. C. Meikap, and M. N. Biswas, "Performance of a modified multi-stage bubble column reactor for lead(ii) and biological oxygen demand removal from wastewater using activated rice husk," Journal of Hazardous Materials, vol. 161, no. 1, pp. 317-324, 2009.

[3] Z. Salehi, H. Yoshikawa, R. Mineta, and Y. Kawase, "Aerobic biodegradation of p-nitrophenol by acclimated waste activated sludge in a slurry bubble column," Process Biochemistry, vol. 46, no. 1, pp. 284-289, 2011.

[4] R. Mineta, Z. Salehi, H. Yoshikawa, and Y. Kawase, "Oxygen transfer during aerobic biodegradation of pollutants in a dense activated sludge slurry bubble column: actual volumetric oxygen transfer coefficient and oxygen uptake rate in p-nitrophenol degradation by acclimated waste activated sludge," Biochemical Engineering Journal, vol. 53, no. 3, pp. 266-274, 2011.

[5] X. F. Liang, H. Pan, Y. H. Su, and Z. H. Luo, "CFD-PBM approach with modified drag model for the gas-liquid flow in a bubble column," Chemical Engineering Research and Design, vol. 112, pp. 88-102, 2016.
[6] S. Besbes, M. E. Hajem, H. B. Aissia, J. Y. Champagne, and J. Jay, "PIV measurements and Eulerian-Lagrangian simulations of the unsteady gas-liquid flow in a needle sparger rectangular bubble column," Chemical Engineering Science, vol. 126, pp. 560-572, 2015.

[7] B. A. Ali, C. S. Kumar, and S. Pushpavanam, "Analysis of liquid circulation in a rectangular tank with a gas source at a corner," Chemical Engineering Journal, vol. 144, pp. 442452, 2008.

[8] B. A. Ali and S. Pushpavanam, "Analysis of unsteady gasliquid flows in a rectangular tank: comparison of EulerEulerian and Euler-Lagrangian simulations," International Journal of Multiphase Flow, vol. 37, no. 3, pp. 268-277, 2011.

[9] T. Wang and J. Wang, "Numerical simulations of gas-liquid mass transfer in bubble columns with a CFD-PBM coupled model," Chemical Engineering Science, vol. 62, no. 24, pp. 7107-7118, 2007.

[10] A. Gupta and S. Roy, "Euler-Euler simulation of bubbly flow in a rectangular bubble column: experimental validation with radioactive particle tracking," Chemical Engineering Journal, vol. 225, pp. 818-836, 2013.

[11] L. Fan, N. Xu, Z. Wang, and H. Shi, "PDA experiments and CFD simulation of a lab-scale oxidation ditch with surface aerators," Chemical Engineering Research and Design, vol. 88, pp. 23-33, 2010.

[12] N. Ratkovich, W. Horn, F. P. Helmus et al., "Activated sludge rheology: a critical review on data collection and modelling," Water Research, vol. 47, p. 463, 2013.

[13] I. Seyssiecq, J. H. Ferrasse, and N. Roche, "State-of-the-art: rheological characterisation of wastewater treatment sludge," Biochemical Engineering Journal, vol. 16, no. 1, pp. 41-56, 2003.

[14] W. D. Deckwer, K. Nguyentien, A. Schumpe, and Y. Serpemen, "Oxygen mass transfer into aerated CMC solutions in a bubble column," Biotechnology and Bioengineering, vol. 24, no. 2, pp. 461-481, 1982.

[15] S. M. Yun, Y. H. Kim, and Y. K. Sang, "Bubble and gas holdup characteristics in a bubble column of CMC solution," Korean Journal of Chemical Engineering, vol. 7, no. 1, pp. 31-39, 1990.

[16] A. Esmaeili, C. Guy, and J. Chaouki, "Local hydrodynamic parameters of bubble column reactors operating with nonNewtonian liquids: experiments and models development," AIChE Journal, vol. 62, no. 4, pp. 1382-1396, 2016.

[17] A. D. Passos, V. P. Voulgaropoulos, S. V. Paras, and A. A. Mouza, "The effect of surfactant addition on the performance of a bubble column containing a non-Newtonian liquid," Chemical Engineering Research and Design, vol. 95, pp. 93-104, 2015.

[18] D. Dapelo, F. Alberini, and J. Bridgeman, "Euler-Lagrange CFD modelling of unconfined gas mixing in anaerobic digestion," Water Research, vol. 85, pp. 497-511, 2015.

[19] T. K. Bandyopadhyay and S. K. Das, "Non-Newtonian and gas-non-Newtonian liquid flow through elbows-CFD analysis," Journal of Applied Fluid Mechanics, vol. 6, pp. 131-141, 2013.

[20] B. X. Wu, "CFD simulation of gas and non-Newtonian fluid two-phase flow in anaerobic digesters," Water Research, vol. 44, pp. 3861-3874, 2010.

[21] S. Li, Y. Ma, S. Jiang, T. Fu, C. Zhu, and H. Z. Li, "The drag coefficient and the shape for a single bubble rising in nonNewtonian fluids," Journal of Fluids Engineering, vol. 134, article 084501, 2012.

[22] M. E. Díaz, A. Iranzo, D. Cuadra, R. Barbero, F. J. Montes, and M. A. Galán, "Numerical simulation of the gas-liquid flow in 
a laboratory scale bubble column: influence of bubble size distribution and non-drag forces," Chemical Engineering Journal, vol. 139, no. 2, pp. 363-379, 2008.

[23] M. Gresch, M. Armbruster, D. Braun, and W. Gujer, "Effects of aeration patterns on the flow field in wastewater aeration tanks," Water Research, vol. 45, no. 2, pp. 810-818, 2011.

[24] D. Zhang, N. G. Deen, and J. A. M. Kuipers, "Numerical simulation of the dynamic flow behavior in a bubble column: a study of closures for turbulence and interface forces," Chemical Engineering Science, vol. 61, no. 23, pp. 7593-7608, 2006.

[25] K. Dewsbury, D. Karamanev, and A. Margaritis, "Hydrodynamic characteristics of free rise of light solid particles and gas bubbles in non-Newtonian liquids," Chemical Engineering Science, vol. 54, no. 21, pp. 4825-4830, 1999.

[26] B. X. Wu and S. L. Chen, "CFD simulation of non-Newtonian fluid flow in anaerobic digesters," Biotechnology and Bioengineering, vol. 99, no. 3, pp. 700-711, 2008.

[27] A. M. Lali, A. S. Khare, J. B. Joshi, and K. D. P. Nigam, "Behaviour of solid particles in viscous non-Newtonian solutions: settling velocity, wall effects and bed expansion in solid-liquid fluidized beds," Powder Technology, vol. 57, no. 1, pp. 39-50, 1989.

[28] J. R. Grace, "Shapes and velocities of single drops and bubbles moving freely through immiscible liquids," Transactions of the Institution of Chemical Engineers, vol. 54, no. 3, pp. 167-173, 1976.

[29] C. Laborde-Boutet, F. Larachi, N. Dromard, O. Delsart, and D. Schweich, "CFD simulation of bubble column flows: investigations on turbulence models in RANS approach," Chemical Engineering Science, vol. 64, no. 21, pp. 4399-4413, 2009.

[30] M. J. Prince and H. W. Blanch, "Bubble coalescence and break-up in air sparged bubble columns," AIChE Journal, vol. 36, no. 10, pp. 1485-1499, 1990.

[31] H. Luo and H. F. Svendsen, "Theoretical model for drop and bubble breakup in turbulent dispersions," Chemical Engineering Science, vol. 66, pp. 766-776, 1996.

[32] D. Ramkrishna, Population Balances: Theory and Applications to Particulate Systems in Engineering, Academic Press, San Diego, CA, USA, 2000.

[33] H. Luo, Coalescence, Breakup and Liquid Circulation in Bubble Column Reactors, Norwegian Institute of Technology, Trondheim, Norway, 1993.

[34] G. Li, X. Yang, and G. Dai, "CFD simulation of effects of the configuration of gas distributors on gas-liquid flow and mixing in a bubble column," Chemical Engineering Science, vol. 64, no. 24, pp. 5104-5116, 2009.

[35] R. M. A. Masood, Y. Khalid, and A. Delgado, "Scale adaptive simulation of bubble column flows," Chemical Engineering Journal, vol. 262, pp. 1126-1136, 2015.

[36] V. V. Buwa and V. V. Ranade, "Dynamics of gas-liquid flow in a rectangular bubble column: experiments and single/ multi-group CFD simulations," Chemical Engineering Science, vol. 57, no. 22-23, pp. 4715-4736, 2002.

[37] C. Durán, Y. Fayolle, Y. Pechaud, A. Cockx, and S. Gillot, "Impact of suspended solids on the activated sludge nonNewtonian behaviour and on oxygen transfer in a bubble column," Chemical Engineering Science, vol. 141, pp. 154-165, 2016.

[38] L. Böhm, T. Kurita, K. Kimura, and M. Kraume, "Rising behaviour of single bubbles in narrow rectangular channels in Newtonian and non-Newtonian liquids," International Journal of Multiphase Flow, vol. 65, pp. 11-23, 2014. 


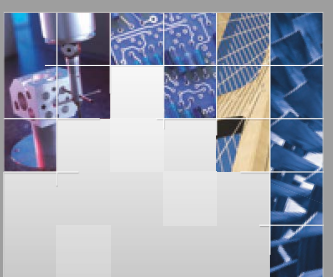

\section{Enfincering}
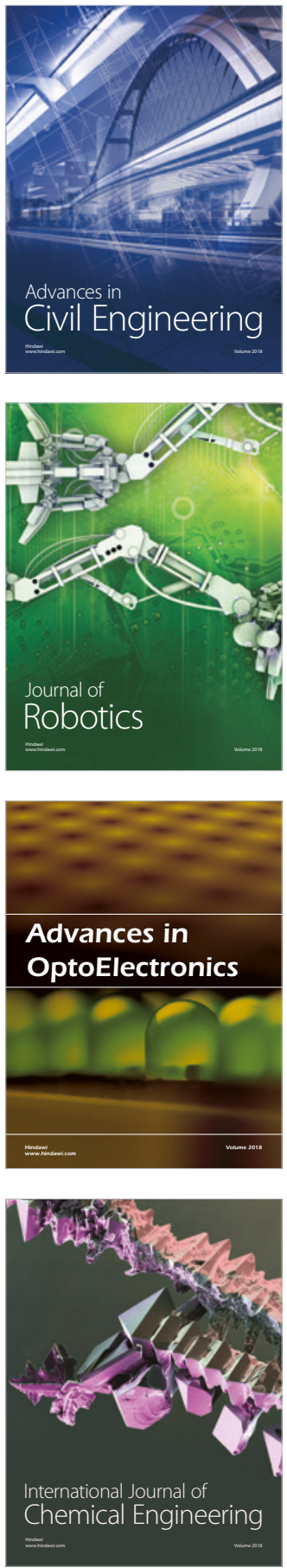



\section{Rotating \\ Machinery}

The Scientific World Journal

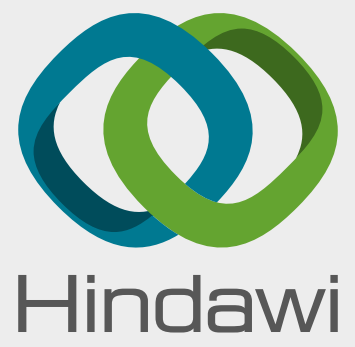

Submit your manuscripts at

www.hindawi.com
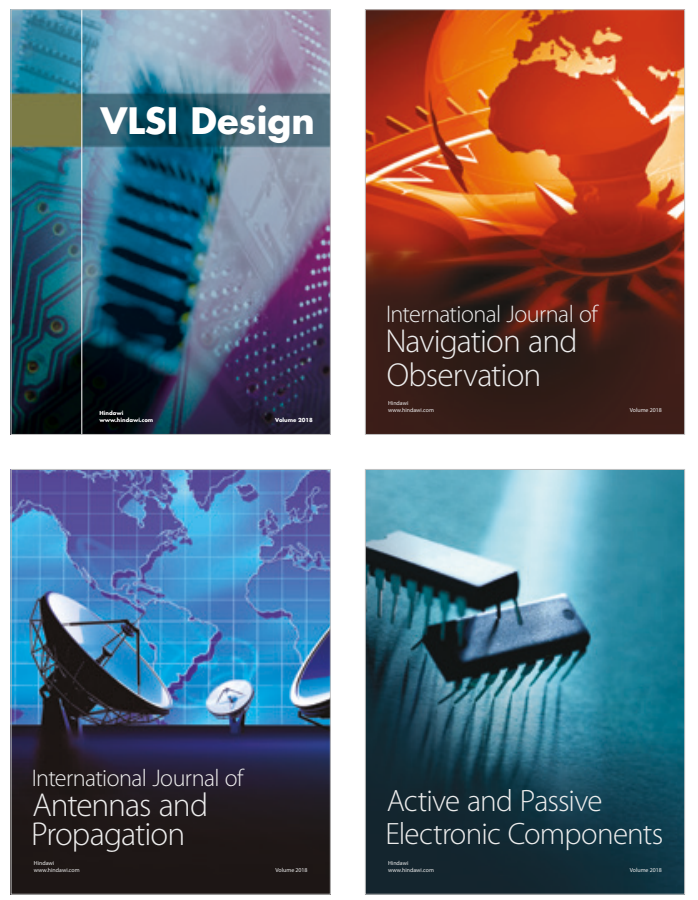
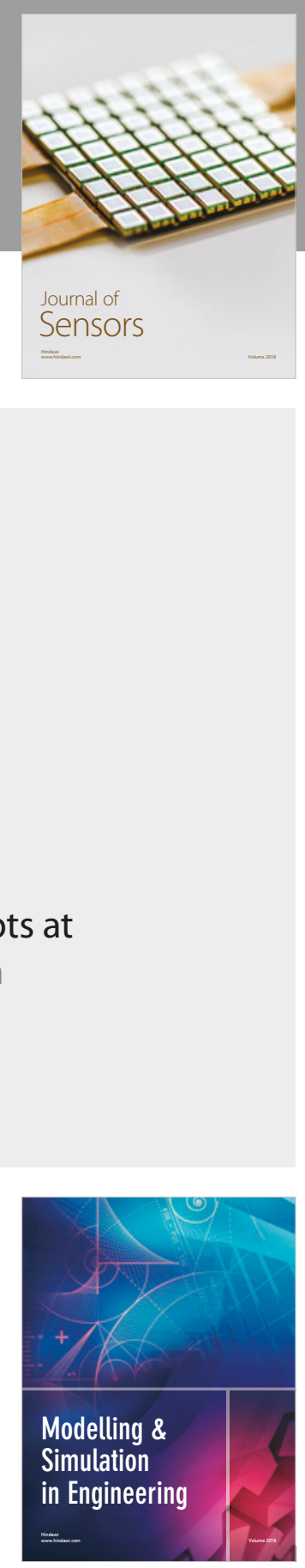

\section{Advances \\ Multimedia}
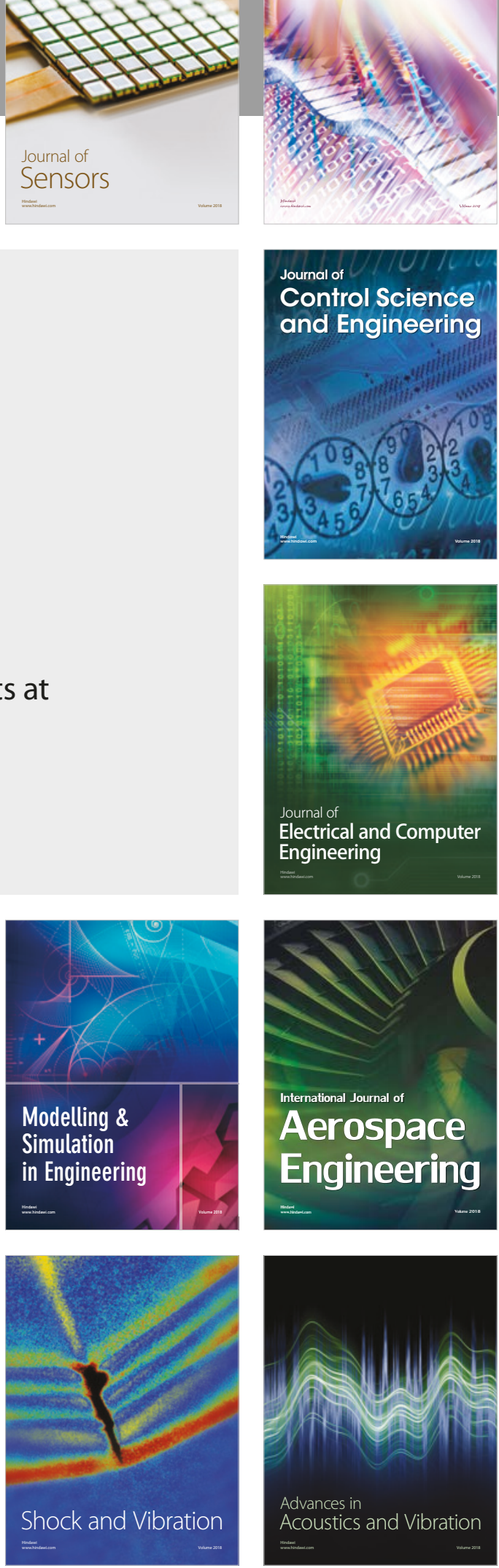